1

2 Analysis of unusual and signature APOBEC-mutations in HIV-1 pol next-generation sequences
4

12 1'Division of Infectious Diseases, Department of Medicine, Stanford University, Stanford, CA, USA;

$13{ }^{2}$ Institute for Genomics and Evolutionary Medicine, Temple University, Philadelphia, PA, USA;

$14{ }^{3}$ Centre for Research in Infectious Diseases, National Institute of Respiratory Diseases, Tlalpan

15 4502, 14080, Mexico City, Mexico; 'Department of Statistics, Stanford University, Stanford, CA,

16 USA; ${ }^{5}$ Division of Infectious Diseases, Brown University Alpert Medical School, 164 Summit

17 Avenue, Providence RI 02906, US

18

\section{CORESSPONDING AUTHORS}

Philip L. Tzou (philiptz@stanford.edu)

Robert W. Shafer (rshafer@stanford.edu) 
bioRxiv preprint doi: https://doi.org/10.1101/831685; this version posted November 5,2019 . The copyright holder for this preprint (which was not certified by peer review) is the author/funder, who has granted bioRxiv a license to display the preprint in perpetuity. It is made available under aCC-BY 4.0 International license.

23 Abstract word count: 300

24 Manuscript word count: 3,253

25 


\section{ABSTRACT}

27 Introduction: At low mutation-detection thresholds, next generation sequencing (NGS) for HIV-

281 genotypic resistance testing is susceptible to artifactual detection of mutations arising from

29 PCR error and APOBEC-mediated G-to-A hypermutation. Methods: We analyzed published HIV-

$301 \mathrm{pol}$ Illumina NGS data to characterize the distribution of mutations at eight NGS thresholds:

$3120 \%, 10 \%, 5 \%, 2 \%, 1 \%, 0.5 \%, 0.2 \%$, and $0.1 \%$. At each threshold, we determined the proportion

32 of amino acid mutations that were unusual (defined as having a prevalence $<0.01 \%$ in HIV-1 group

33 M sequences) or were signature APOBEC mutations. Results: Eight studies, containing 855

34 samples, in the NCBI Sequence Read Archive were analyzed. As detection thresholds were

35 lowered, there was a progressive increase in the proportion of positions with both usual and

36 unusual mutations and in the proportion of all mutations that were unusual. The median

37 proportion of positions with an unusual mutation increased gradually from $0 \%$ at the $20 \%$

38 threshold to $0.3 \%$ at the $1 \%$ threshold and then exponentially to $1.3 \%$ ( $0.5 \%$ threshold), $6.9 \%$

39 (0.2\% threshold), and $23.2 \%$ (0.1\% threshold). In two of three studies with available plasma HIV-1

RNA levels, the proportion of positions with unusual mutations was negatively associated with

41 virus levels. Although the complete set of signature APOBEC mutations was much smaller than

42 that of unusual mutations, the former outnumbered the latter in one-sixth of the samples at the

$430.5 \%, 1 \%$, and $2 \%$ thresholds. Conclusions: The marked increase in the proportion of positions

44 with unusual mutations at thresholds below $1 \%$ and in samples with lower virus loads suggests

45 that, at low thresholds, many unusual mutations are artifactual, reflecting PCR error or G-to-A

46 hypermutation. Profiling the numbers of unusual and signature APOBEC pol mutations at 
bioRxiv preprint doi: https://doi.org/10.1101/831685; this version posted November 5,2019 . The copyright holder for this preprint (which was not certified by peer review) is the author/funder, who has granted bioRxiv a license to display the preprint in perpetuity. It is made available under aCC-BY 4.0 International license.

47 different NGS thresholds may be useful to avoid selecting a threshold that is too low and poses

48 an unacceptable risk of identifying artifactual mutations.

49 


\section{INTRODUCTION}

Next-generation sequencing (NGS) is increasingly performed for HIV-1 genotypic

52 resistance testing [1]. However, low levels of plasma viremia and/or inefficient RNA extraction,

53 or reverse transcription may result in a low number of amplifiable cDNA templates. In such

54 scenarios, much of the observed variability in an NGS sequence may reflect PCR error rather than

55 authentic viral mutations [2-4]. Since PCR errors are not subject to selective forces exerted during

56 virus evolution, we have hypothesized that the presence of large numbers of unusual and likely

57 deleterious mutations at an NGS mutation detection threshold suggests the threshold is too low

$58[5-8]$

59 NGS is also more likely than Sanger sequencing to detect low frequency APOBEC-

60 mediated G-to-A hypermutation [9-11]. APOBEC-mediated G-to-A hypermutation can be

61 detected if plasma samples are contaminated with proviral DNA templates, which are enriched

62 for defective viruses [12], or if defective hypermutated virus genomes are successfully packaged

63 and released from cells. Hypermutated viruses are unlikely to be functional because they often

64 contain premature stop codons and mutations at highly conserved residues [9-11, 13]. Therefore,

65 the detection of drug-resistance mutations (DRMs) that could be caused by APOBEC in viruses

66 with evidence for G-to-A hypermutation has questionable clinical significance.

67 In this study, we systematically analyze HIV-1 pol NGS data from eight published studies

68 to characterize the distribution of unusual mutations and mutations suggestive of APOBEC-

69 mediated G-to-A hypermutation at different NGS thresholds.

70

71

\section{METHODS}


72 NGS datasets, FASTQ files, and codon frequency tables

We searched the NCBI Sequence Read Archive BioProject Library and other public

74 repositories to identify NGS data sets of HIV-1 pol meeting the following criteria: (i) sequencing

75 was performed on Illumina instruments; (ii) samples contained at least 10 clinical specimens; (iii)

76 samples were from plasma HIV-1 RNA rather than proviral DNA; and (iv) samples required PCR

77 amplification (i.e., were not from metagenomic studies). The publications associated with these

78 datasets were reviewed to retrieve the following information for each sample: plasma HIV-1 RNA

79 level, volume of plasma submitted for RNA extraction, number of sequencing reads, and methods

80 of RNA extraction, RT-PCR, and library preparation. Datasets for which there was no associated

81 publication were excluded (Figure 1).

additional study was identified in the research upload site Zenodo
FASTQ file. Briefly, we filtered reads with fewer than 100 nucleotides or a mean quality (phred or

91 remaining reads were used to populate a codon frequency table with five fields: 
3. Number of reads for the position (coverage).

95 4. Sequenced codon.

5. Number of reads containing the codon. insertion [indicated by more than one amino acid], a deletion, or a frame shift if the codon does not contain a multiple of three bases).

2. The fraction of reads containing the codon.

3. The prevalence of the corresponding amino acid in group $\mathrm{M}$ sequences in HIVDB.

4. The prevalence of the codon in group $M$ sequences in HIVDB.

5. Whether the amino acid encodes a drug-resistance mutation (DRM).

6. Whether the amino acid is a signature APOBEC mutation.

7. Whether the amino acid is a DRM that could also be caused by APOBEC. interpretation program to impute its subtype.

Unusual mutations

112 in a GitHub repository (https://github.com/hivdb/hivfacts). The group M mutation prevalence

113 data was derived from direct PCR ("population based") plasma virus Sanger sequences in HIVDB,

114 from which poor-quality sequences had been filtered. The prevalence of an amino acid at a 
116 the total number of sequences containing the position. Each mutation was counted once per

117 individual. Unusual mutations were defined as mutations with a group $\mathrm{M}$ prevalence $<0.01 \%$ and

118 not on the list of known DRMs (i.e., without an HIVDB mutation penalty score).

119 Overall, 4,651 (23.4\%) of the 19,887 possible amino acids in the 947 protease, RT, and

120 integrase positions were classified as usual and 15,236 (76.6\%) were classified as unusual. The

121 usual amino acids included the 947 (20.4\%) amino acids constituting the Los Alamos National

122 Laboratories group $M$ consensus [16] and 3,704 non-consensus amino acids. The 3,704 non-

123 consensus usual amino acids included 2,750 (74.2\%) with one nucleotide difference from the

124 group $M$ consensus, 867 (23.4\%) with two nucleotide differences, and 87 (2.3\%) with three

125 nucleotide differences. Among the unusual mutations, 3,133 (20.6\%) had one nucleotide

126 difference from the group M consensus, 8,503 (55.8\%) had two nucleotide differences, and 3,600

127 (23.6\%) had three nucleotide differences.

128 Pearson correlation coefficient $(r)$ was used to quantify the association between a 129 sample's (i) virus load and proportion of positions with usual or unusual mutations; and (ii) 130 median number of sequence reads per position and proportion of positions with usual or unusual

131 mutations.

Signature APOBEC mutations

Amino acid mutations consistent with APOBEC-mediated G-to-A hypermutation were

135 identified using a modification of a previously described procedure [6]. Briefly, we found all

136 positions in PR, RT, and IN for which $>50 \%$ of amino acids at a position represented a potential

137 target for APOBEC3F (5'GA dinucleotides) or APOBEC3G (5'GG dinucleotides). We then identified 
138 all amino acid mutations that would result from APOBEC3F or $3 G$ editing of these potential

139 targets. Each of the resulting APOBEC-context mutations were then examined for their

140 prevalence in group $M$ sequences and for their association with stop codons or active site

141 mutations. Stop codons result from APOBEC3G editing of tryptophan (W): TGG $\rightarrow$ TAG or TGG $\rightarrow$

142 TGA, when tryptophan is followed by an amino acid beginning with an A or G. Active site

143 mutations in PR (D25N), RT (D110N, D185N, and D186N), and IN (D64N, D116N, and E152K) result

144 from APOBEC3F editing of aspartic acid GAC/T (D) $\rightarrow$ AAC/T (N) or glutamic acid GAA/G $(E) \rightarrow$

$145 \quad A A A / G(K)$.

APOBEC-context mutations that met the following criteria were considered signature

147 APOBEC mutations: (i) they occurred at a prevalence $<0.1 \%$ or at a prevalence $<0.5 \%$ if they

148 occurred frequently in sequences with stop codons or active site mutations and (ii) they were not

149 known DRMs. Overall, we identified 296 signature APOBEC mutations. Based on a previous study

150 [6] and a comparison with the LANL Hypermut program [17], we determined that pol genes

151 containing three or more signature APOBEC mutations in were likely to have undergone APOBEC-

152 mediated G-to-A hypermutation (Supplementary text).

153 Overall, 175 (59.1\%) of the 296 signature APOBEC mutations were also unusual (i.e.,

154 prevalence $<0.01 \%)$. The remaining signature APOBEC mutations, which had a prevalence

155 ranging from $0.01 \%$ to $0.4 \%$, were classified based on their genetic context, their rarity, and their

156 strong association with stop codons and active site mutations. In contrast, just $1.2 \%$ of the 15,236

157 unusual mutations were also signature APOBEC mutations. 


\section{$160 \quad$ NGS datasets}

162 (Figure 1; Table 1). These samples included 693 PR, 700 RT, and 449 IN NGS sequence sets. Of the

163 RT samples, 209 encompassed all 560 amino acid positions. Ninety percent of the remaining

164 samples encompassed at least the first 240 amino acid positions. Subtype B accounted for 606

165 (70.9\%) of samples. Subtypes A, C, CRF01_AE, and CRF02_AG were the most common non-B

166 subtypes, accounting for 224 (26.2\%) of sequences. Plasma HIV-1 RNA levels were available for

167 all sequenced samples in three studies $[21,23,24]$. 


\begin{tabular}{|c|c|c|c|c|c|c|c|}
\hline $\begin{array}{l}\text { Author } \\
\text { (Yr) }\end{array}$ & Title & $\begin{array}{c}\# \\
\text { Samples }^{1}\end{array}$ & Genes & $V L^{2}$ & Region & $\begin{array}{l}\text { ARV } \\
\text { Status }\end{array}$ & Subtypes $^{3}$ \\
\hline $\begin{array}{l}\text { Avila-Rios } \\
\text { (2016)[23] }\end{array}$ & $\begin{array}{l}\text { HIV Drug Resistance in Antiretroviral } \\
\text { Treatment-Naïve Individuals in the Largest } \\
\text { Public Hospital in Nicaragua, 2011-2015 }\end{array}$ & 255 & $\mathrm{PR} / \mathrm{RT}$ & Yes & Nicaragua & Naive & B (99.6\%) \\
\hline $\begin{array}{l}\text { Moscona } \\
\text { (2017)[19] }\end{array}$ & $\begin{array}{l}\text { Comparison between next-generation and Sanger- } \\
\text { based sequencing for the detection of transmitted } \\
\text { drug-resistance mutations among recently infected } \\
\text { HIV-1 patients in Israel, 2000-2014 }\end{array}$ & 78 & $\begin{array}{l}\text { PR (76); RT } \\
\text { (77); IN (30) }\end{array}$ & No & Israel & Naïve & $\begin{array}{l}\text { B (59\%); C (22\%); A } \\
(14 \%)\end{array}$ \\
\hline $\begin{array}{l}\text { Huber } \\
\text { (2016)[24] }\end{array}$ & $\begin{array}{l}\text { MinVar: A rapid and versatile tool for HIV-1 drug } \\
\text { resistance genotyping by deep sequencing }\end{array}$ & 33 & $\begin{array}{l}\mathrm{PR} / \mathrm{RT}(33) ; \\
\text { IN (13) }\end{array}$ & Yes & Switzerland & NA & $\begin{array}{l}\text { B (67\%), A (9\%), C (9\%), } \\
\text { CRF02_AG (9\%) }\end{array}$ \\
\hline $\begin{array}{l}\text { Nguyen } \\
\text { (2018)[20] }\end{array}$ & $\begin{array}{l}\text { Prevalence and clinical impact of minority resistant } \\
\text { variants in patients failing an integrase inhibitor- } \\
\text { based regimen by ultra-deep sequencing }\end{array}$ & 134 & IN & No & France & Treated & $\begin{array}{l}\text { B (60\%), CRF02_AG } \\
(26 \%)\end{array}$ \\
\hline $\begin{array}{l}\text { Dalmat } \\
\text { (2018)[25] }\end{array}$ & $\begin{array}{l}\text { Limited marginal utility of deep sequencing for HIV } \\
\text { drug resistance testing in the age of integrase } \\
\text { inhibitors }\end{array}$ & 112 & $\begin{array}{l}\text { PR (93); RT } \\
\text { (94); IN (38) }\end{array}$ & No & U.S. & Treated & B (95\%), C (4\%), D (1\%) \\
\hline
\end{tabular}




\begin{tabular}{|c|c|c|c|c|c|c|c|}
\hline $\begin{array}{l}\text { Jair } \\
\text { (2019)[18] }\end{array}$ & $\begin{array}{l}\text { Validation of publicly-available software used in } \\
\text { analyzing NGS data for HIV-1 drug resistance } \\
\text { mutations and transmission networks in a } \\
\text { Washington, DC, cohort. }\end{array}$ & 42 & $\begin{array}{l}\text { PR (34), RT } \\
(41), \text { IN (33) }\end{array}$ & No & U.S. & Treated & B (98\%), CRF02_AG (2\%) \\
\hline $\begin{array}{l}\text { Ode } \\
\text { (2015)[21] }\end{array}$ & $\begin{array}{l}\text { Quasispecies Analyses of the HIV-1 Near-full-length } \\
\text { Genome With Illumina MiSeq }\end{array}$ & 92 & $\mathrm{PR} / \mathrm{RT} / \mathrm{IN}$ & Yes & Japan & Treated & $\begin{array}{l}\text { B (61\%), CRF01_AE } \\
(11 \%), C(11 \%), \text { CRF02 } \\
(9 \%)\end{array}$ \\
\hline $\begin{array}{l}\text { Telele } \\
\text { (2019)[22] }\end{array}$ & $\begin{array}{l}\text { Pretreatment drug resistance in a large countrywide } \\
\text { Ethiopian HIV-1C cohort: a comparison of Sanger and } \\
\text { high-throughput sequencing. }\end{array}$ & 109 & $\mathrm{PR} / \mathrm{RT} / \mathrm{IN}$ & No & Ethiopia & Naive & C (99\%), D (1\%) \\
\hline \multicolumn{8}{|c|}{$\begin{array}{l}{ }^{1} \text { Ode } 2015 \text { contained } 92 \text { samples from } 58 \text { persons. }{ }^{2} \text { Virus load (VL) data was available for all se } \\
\text { available for a small subset of patients. }{ }^{3} \text { Samples with uncommon subtypes are not shown. }\end{array}$} \\
\hline
\end{tabular}


bioRxiv preprint doi: https://doi.org/10.1101/831685; this version posted November 5, 2019. The copyright holder for this preprint (which was not certified by peer review) is the author/funder, who has granted bioRxiv a license to display the preprint in perpetuity. It is made available under aCC-BY 4.0 International license.

$1711.0 \mathrm{ml}$ of plasma, high-fidelity RT and PCR enzymes, and nested PCR. However, the specific

172 extraction protocols and enzymes used for PCR amplification varied. Amplicon sizes also varied

173 from 750 to $4,400 \mathrm{bp}$. Across all studies, the median coverage per position was 18,275 , with a $5 \%$

174 to $95 \%$ range of 2,944 to 81,184 . 


\begin{tabular}{|c|c|c|c|c|c|}
\hline Author (Yr) & RNA Extraction ${ }^{1}$ & RT / PCR Enzymes ${ }^{1}$ & $\begin{array}{l}\text { PCR Product } \\
\text { Size }(b p)^{2}\end{array}$ & $\begin{array}{l}\text { Sequence Length } \\
\text { (5\% to } 95 \% \text { Range) }\end{array}$ & $\begin{array}{l}\text { Median \# Reads } \\
\text { (5\% to } 95 \% \text { Range) }\end{array}$ \\
\hline Avila-Rios (2016) & $\begin{array}{l}\text { QIAamp Viral RNA Mini Kit } \\
\text { (1 ml plasma) }\end{array}$ & $\begin{array}{l}\text { SuperScript III OneStep RT PCR followed by } \\
\text { Platinum Taq DNA polymerase }\end{array}$ & 1,592 & $436(432-440)$ & $\begin{array}{c}30,079 \\
(10,721-53,845)\end{array}$ \\
\hline Moscona (2017) & $\begin{array}{c}\text { NucliSENS easyMAG (500 ul } \\
\text { plasma) }\end{array}$ & NA & 1,800 & $436(313-603)$ & $\begin{array}{c}5,652 \\
(1,306-11,359)\end{array}$ \\
\hline Huber (2016) & $\begin{array}{c}\text { NucliSENS easyMAG } \\
\text { (500 ul plasma) }\end{array}$ & $\begin{array}{l}\text { PrimeScript One-Step RT PCR Kit followed } \\
\text { by Phusion HotStart II HF polymerase }\end{array}$ & 3,500 & $440(423-947)$ & $\begin{array}{c}72,712 \\
(11,486-107,307)\end{array}$ \\
\hline Nguyen (2018) & $\begin{array}{c}\text { NucliSENS easyMAG } \\
\text { (1 } \mathrm{ml} \text { plasma) }\end{array}$ & $\begin{array}{l}\text { Transcriptor One-Step RT-PCR followed by } \\
\text { QS High Fidelity PCR Kit }\end{array}$ & 763 & $232(227-235)$ & $\begin{array}{c}13,878 \\
(8,788-54,314)\end{array}$ \\
\hline Dalmat (2018) & $\begin{array}{l}\text { Boom silica } \\
\text { (400 ul plasma) }\end{array}$ & GeneAmp RNA PCR Kit & $\begin{array}{c}1,306(\mathrm{PR} / \mathrm{RT}) \\
1,306(\mathrm{IN})\end{array}$ & $358(318-713)$ & $\begin{array}{c}5,718 \\
(1,781-17,979)\end{array}$ \\
\hline Jair (2019) & QIAamp Viral RNA Mini Kit & HiFi Taq DNA polymerase & $883(\mathrm{PR}), 652$ & $669(228-771)$ & 41,833 \\
\hline
\end{tabular}




\begin{tabular}{|c|c|c|c|c|c|}
\hline & (150 ul plasma) & & $\begin{array}{l}\text { (RT), } 1000+ \\
\text { (IN) }\end{array}$ & & $(2,681-131,625)$ \\
\hline Ode (2015) & $\begin{array}{l}\text { Magnapure compact NA } \\
\text { isolation kit } \\
\text { (200-400 ul plasma) }\end{array}$ & $\begin{array}{l}\text { PrimeScript I high Fidelity One Step RT-PCR } \\
\text { Kit followed by PrimeSTAR GXL DNA } \\
\text { Polymerase }\end{array}$ & 2,700 & 947 (947-947) & $\begin{array}{c}13,322 \\
(4,453-30,279)\end{array}$ \\
\hline Telele (2019) & $\begin{array}{l}\text { QIAamp Viral RNA Mini Kit } \\
\qquad(150 \text { ul plasma })\end{array}$ & HiFi Taq DNA polymerase & 4,360 & $947(947-947)$ & $\begin{array}{c}31,288 \\
(16,467-96,237)\end{array}$ \\
\hline \multicolumn{6}{|c|}{$\begin{array}{l}\text { Footnote: }{ }^{1} \text { Reagent manufacturers: QIAamp Viral RNA Mini Kit (QIAGEN); NucliSENS easyMAG (bioMerieux Clinical Diagnostics); Magnapure compact NA } \\
\text { isolation kit (Roche Life Sciences); SuperScript III OneStep RT PCR (Invitrogen); PrimeScript One-Step RT PCR Kit (Takara, Kusatsu, Japan); Phusion HotStart II } \\
\text { HF polymerse (ThermoFisher); Transcriptor One-Step RT-PCR (Roche); QS High Fidelity PCR Kit (New England Biolabs); GeneAmp RNA PCR Kit (Perkin-Elmer); } \\
\text { HiFi Taq DNA polymerase (Takara; Mountain View, CA, US). For Ode 2015, products from three separate PCR reactions were pooled. }{ }^{2} \text { PCR product sizes were } \\
\text { estimated from the HXB2 coordinates provided for the first round of PCR. For Jair 2019, it was not possible to precisely determine the size of the integrase (IN) }\end{array}$} \\
\hline
\end{tabular}


177 Usual and unusual mutations at different NGS thresholds

178

179

180

181

182

183

184

185

\section{$\underline{\text { Pooled data from all datasets }}$}

Figure 2 depicts the proportion of positions with usual (panel A) and unusual (panel B) mutations and the proportion of all mutations that were unusual (number of unusual mutations / total number of mutations, panel C) as a function of detection threshold in pooled samples for all studies.

\section{Figure 2. Boxplots demonstrating the distribution in the the proportion of positions with}

$$
\text { usual mutations }(A) \text {, the proportion of positions with unusual mutations }(B) \text {, and the }
$$$$
\text { proportion of mutations that were unusual (number of unusual mutations / [number of }
$$

$$
\text { usual mutations + number of unusual mutations]) (C) at eight NGS thresholds for pooled }
$$

samples $(n=855)$ from eight published studies.

The median proportion of positions with a usual mutation increased from $5.2 \%$ to $11.6 \%$

between the $20 \%$ and $0.5 \%$ thresholds then began doubling to $23.6 \%$ at the $0.2 \%$ threshold and to $47.2 \%$ at the $0.1 \%$ thresholds.

The median proportion of positions with an unusual mutation increased from $0 \%$ to $0.3 \%$

between the $20 \%$ and $1 \%$ thresholds but then began increasing about four-fold to $1.3 \%$ at the $0.5 \%$ threshold, $6.9 \%$ at the $0.2 \%$ threshold, and $23.2 \%$ at the $0.1 \%$ thresholds.

The median proportion of mutations that were unusual (number of unusual mutations /

[number of usual mutations + number of unusual mutations]) increased from $0 \%$ to $1.1 \%$ between the $20 \%$ and $2 \%$ threshold but then jumped to $4.2 \%$ at the $1 \%$ threshold, $12.0 \%$ at the $0.5 \%$ threshold, and to $25.1 \%$, and $33.9 \%$ respectively at the $0.2 \%$ and $0.1 \%$ thresholds. 
There was a weak but statistically significant relationship between the $\log _{10}$ of the number of sequence reads (i.e., coverage) and the number of usual mutations (correlation coefficient $r$ between 0.21 and $0.24, p<0.001$ ) at the $1 \%, 2 \%, 5 \%, 10 \%$, and $20 \%$ thresholds (Supplementary Figure 1). The relationship between the number of sequence reads and the number of unusual mutations was much weaker with $r$ between 0.13 and 0.17 at the $1 \%, 2 \%$, and $5 \%$ thresholds (Supplementary Figure 2).

Figures $3 \mathrm{~A}$ and $3 \mathrm{~B}$ plot the median proportion of positions with usual and unusual

207 mutations, respectively, at the eight NGS thresholds for pooled samples within each of the eight studies. Figure $3 \mathrm{C}$ plots the median proportion of mutations that were unusual at each threshold for each study. These figures indicate that there were clear interstudy differences in the

210 distributions of usual and unusual mutations, particularly at thresholds below 1\%. The eight

211 studies visually clustered into three groups at the three lowest thresholds. Two studies had the

212 lowest numbers of both usual and unusual mutations, and of the proportions of unusual

213 mutations [21, 22]. Four studies had intermediate values for these three metrics [18, 20, 24, 25],

214 and two had higher values [19, 23]. 
One of the two studies with the lowest numbers of unusual mutations pooled PCR

221 product from three separate reactions, potentially lowering the impact of artifacts propagated

222 per reaction [21]. However, because of the many inter-study differences in sample characteristics

223 and laboratory procedures, we were otherwise unable to assess whether specific sample

224 characteristics or laboratory procedures were responsible for observed differences in the

225 proportion of positions with usual and unusual mutations.

226 There was marked heterogeneity in the distribution of unusual mutations at different

227 thresholds within each study (Supplementary Figure 3). For example, at the 1\% threshold, the

228 highest number of unusual mutations in each study was generally about five times higher than

229 the median number of unusual mutations.

\section{Association of unusual mutations with virus load}

Plasma HIV-1 RNA levels were available for samples from three of the eight studies [21,

$23323,24]$. The percent of positions with unusual mutations was significantly higher in samples with

234 lower virus loads in two of the three studies $[23,24]$, particularly at the $0.5 \%, 1 \%, 2 \%$, and $5 \%$

235 thresholds (Figures 4A, 4B). This relationship was not detectable either at the very low thresholds

236 of $0.1 \%$ and $0.2 \%$, which contained high numbers of both usual and unusual mutations, or at the

$23710 \%$ and $20 \%$ thresholds, which contained few unusual mutations. There was generally no

238 significant relationship between the proportion of positions with usual mutations and the

239 sample's virus load (Supplementary Figure 4). 
NGS threshold in two of the three studies for which virus load data were available [21,

Figure 5 shows the proportion of positions with signature APOBEC mutations at each

248 threshold. At the $20 \%, 10 \%, 5 \%, 2 \%$, and $1 \%$ thresholds, the median proportion of positions with a signature $A P O B E C$ mutation was 0 . However, at the $2 \%$ and $1 \%$ thresholds, 46 and 113 samples, respectively, contained three or more signature APOBEC mutations; given the average sequence

251 length, this corresponds to approximately $0.9 \%$ of positions.

$257(n=14,940)$, signature APOBEC mutations outnumbered non-APOBEC unusual mutations in

$25816.0 \%, 17.3 \%$, and $13.2 \%$ of samples at the $0.5 \%, 1 \%$, and $2 \%$ thresholds, but in just $2.8 \%$ to $7.5 \%$

259 of samples at the remaining thresholds (Figure 6). 


\section{DISCUSSION}

Through a meta-analysis of NGS pol data from 855 samples in eight published studies, we

267 found that as the mutation detection threshold was lowered, there was a progressive increase in

268 the proportion of sequence positions with both usual and unusual mutations and in the

269 proportion of mutations that are unusual. The proportion of positions with unusual mutations

270 increased gradually from $0 \%$ to $0.3 \%$ between the $20 \%$ and $1 \%$ thresholds and then exponentially

271 to $1.3 \%, 6.9 \%$, and $23.2 \%$ at the $0.5 \%, 0.2 \%$, and $0.1 \%$ thresholds, respectively. Similarly, the

272 proportion of mutations that were unusual increased gradually from $0 \%$ to $1.1 \%$ between the

$27320 \%$ and $2 \%$ threshold but then exponentially to $4.2 \%, 12.0 \%$, and $25.1 \%$, at the $1 \%, 0.5 \%$, and

$274 \quad 0.2 \%$ thresholds, respectively.

275 The marked increase in the proportion of positions with unusual mutations and in the

276 proportion of mutations that were unusual at detection thresholds below $1 \%$ suggests that many

277 of the mutations at low thresholds resulted from processes other than virus replication such as

278 PCR error and APOBEC-mediated hypermutation. Moreover, in two of the studies for which

279 plasma HIV-1 RNA levels were available, the proportion of positions with unusual mutations was

280 inversely related to virus levels at the $0.5 \%, 1 \%, 2 \%$, and $5 \%$ thresholds. A plausible explanation

281 for this pattern is that samples with lower virus loads yield fewer cDNA molecules and that, in

282 these samples, a greater amount of sequence variation results from PCR amplification rather than

283 from HIV-1 replication.

Illumina sequence errors were also likely to have contributed to sequence artifact but 
required to prevent random machine errors from being detected at low thresholds. Indeed, over

287 the complete dataset, the median coverage per position was 18,275 and $95 \%$ of positions had a coverage of nearly 3,000 reads. Thus for $95 \%$ of samples, machine error would have required the same random error to occur at least three times to result in detectable sequence artifacts at the coverage was not correlated with the proportion of positions with unusual mutations also supports the conclusion that most unusual mutations did not result from machine error. sequences even when PCR errors are excluded through the use of unique molecular identifiers

295 (UMIs) [9]. This study indicates that, at the thresholds of $0.5 \%, 1 \%$, and $2 \%$, signature APOBEC mutations outnumber non-APOBEC unusual mutations in approximately one-sixth of samples

297 even though non-APOBEC unusual mutations are far more numerous than signature APOBEC 298 mutations. There are 17 DRMs that could be caused by APOBEC-mediated G-to-A hypermutation: 299 D30N, M46I, G48S, and G73S in PR, D67N, E138K, M184I, G190ES, and M230I in RT, and G118R, E138K, G140S, G163KR, D232N, and R263K in IN. These mutations should be considered possible 301 artifacts if they occur at the same threshold at which multiple signature APOBEC mutations are 302 also present.

To estimate the proportions of positions with unusual mutations generated during HIV-1

304 replication in vivo, we recently performed a meta-analysis of publicly available pol single genome 305 sequences (SGSs) - which are not subject to PCR error - in plasma samples from persons with 306 active HIV-1 replication [8]. We found that in samples with a median of 20 SGSs, the proportion 307 of sequence positions with an unusual mutation was $\leq 1 \%$ in $90 \%$ of samples and $\leq 3 \%$ in $99 \%$ of 
samples. Similarly, the proportion of all mutations that were unusual was $\leq 15 \%$ in $90 \%$ of samples and $\leq 33 \%$ in $99 \%$ of samples. Although the proportion of positions with an unusual mutation might have been higher had the median number of SGSs been $>20$, the proportion of mutations

311 that were unusual would not be affected by the median number of SGSs. Additionally, the

312 estimates of the expected number of unusual mutations based on these SGS data are likely to be

313 inflated as SGSs are subject to errors when RNA is reversed transcribed to cDNA.

The problem of PCR error, as well as the problems of PCR recombination and biased amplification, have led to an approach in which UMIs are added to each cDNA molecule during reverse transcription [26]. Following PCR, consensus sequences are created from reads having

317 the same random ID, making it possible to identify and exclude these errors, which present as 318 mutations that differ from the consensus of sequences containing the same UMI. This approach 319 also has the advantage of providing an estimate of the number of successfully amplified cDNA 320 molecules and of identifying non-consensus sequences that result from PCR recombination. The 321 use of UMIs is becoming the standard approach for investigating HIV-1 dynamics in vivo.

322 However, the use of UMIs necessitates multiple RT reactions and PCR amplifications to generate 323 amplicons spanning HIV-1 drug targets, thus increasing both expense and complexity for 324 genotypic resistance testing for routine clinical or surveillance testing. Several other approaches can also reduce the risk of PCR errors or the likelihood that such errors will lead to artifactual results. First, PCR errors can be reduced by using high fidelity PCR

327 enzymes, although such enzymes often have reduced processivity [27]. Second, the number of 328 PCR cycles may be reduced if a sufficient number of genomic templates are present prior to PCR. 
330 constituent mutation, although such co-occurrences can only be observed over the short genetic

331 distances because most sequence reads are short [28]. Although laboratories often estimate the

332 probability of PCR errors by sequencing plasmid controls, PCR error rates determined by this

333 approach are often underestimates, particularly on samples from which only a small number of

334 cDNA molecules can be generated, as these samples will undergo more PCR amplification than

335 plasmid DNA controls.

336 In addition to applying one or more of the approaches described in the previous

337 paragraphs, we propose that a post hoc analysis of HIV-1 pol NGS data that quantifies the

338 numbers of unusual and signature APOBEC mutations at different NGS thresholds can be useful

339 to avoid selecting a threshold that is too low and that poses an unacceptable risk of identifying

340 artifactual mutations. Such an analysis, demonstrates how the body of published sequence data

341 for a gene (i.e., the prevalence of all mutations at all positions), can be used to optimize the

342 analysis of a new sequence of that gene. To this end, we created a version of the HIVDB genotypic

343 resistance interpretation program that accepts HIV-1 pol codon frequency tables and provides

344 counts of unusual and signature APOBEC mutations and genotypic resistance interpretations at

345 user-selected mutation detection thresholds (https://hivdb.stanford.edu/hivdb/by-reads/). 
SUPPORTING INFORMATION

351 Supplementary Figure 1

352 Scatter plots demonstrating the relationship between log 10 sequence reads and the proportion

353 of positions with usual mutations. Each plot contains the Pearson correlation coefficient $(r)$ and

354 its associated $p$ value.

356 Supplementary Figure 2

357 Scatter plots demonstrating the relationship between log 10 sequence reads and the proportion

358 of positions with unusual mutations. Each plot contains the Pearson correlation coefficient ( $r$ )

359 and its associated $p$ value.

361 Supplementary Figure 3

362 Median and 95\% confidence intervals for the proportion of positions with unusual mutations at

363 eight NGS thresholds for each of the eight studies: A [23], B [19], C [24], D [20], E [25], F [18], G 364 [21], H [22].

366 Supplementary Figure 4

367 Scatter plot demonstrating the relationship between virus load (plasma HIV-1 RNA log copies/ml) 368 and the proportion of positions with usual mutations at four NGS threshold in two of the three 369 studies for which virus load data were available. 


\section{REFERENCES}

373 1. Noguera-Julian M, Edgil D, Harrigan PR, Sandstrom P, Godfrey C, Paredes R. Next-Generation

374 Human Immunodeficiency Virus Sequencing for Patient Management and Drug Resistance

375 Surveillance. J Infect Dis 2017.

376 2. Orton RJ, Wright CF, Morelli MJ, et al. Distinguishing low frequency mutations from RT-PCR

377 and sequence errors in viral deep sequencing data. BMC Genomics 2015; 16:229.

378 3. Gianella S, Delport W, Pacold ME, et al. Detection of minority resistance during early HIV-1

379 infection: natural variation and spurious detection rather than transmission and evolution of

380 multiple viral variants. J Virol 2011; 85:8359-67.

381 4. Learn GH, Jr., Korber BT, Foley B, Hahn BH, Wolinsky SM, Mullins JI. Maintaining the integrity

382 of human immunodeficiency virus sequence databases. J Virol 1996; 70:5720-30.

383 5. Babrzadeh F, Varghese V, Pacold M, et al. Collinearity of protease mutations in HIV-1 samples

384 with high-level protease inhibitor class resistance. J Antimicrob Chemother 2013; 68:414-8.

385 6. Rhee SY, Sankaran K, Varghese V, et al. HIV-1 Protease, Reverse Transcriptase, and Integrase

386 Variation. J Virol 2016; 90:6058-70.

387 7. Tzou PL, Ariyaratne P, Varghese V, et al. Comparison of an In Vitro Diagnostic Next-

388 Generation Sequencing Assay with Sanger Sequencing for HIV-1 Genotypic Resistance Testing. J

389 Clin Microbiol 2018; 56.

390 8. Tzou PL, Rhee SY, Shafer R. Amino Acid Prevalence of HIV-1 pol Mutations by Direct PCR and

391 Single Genome Sequencing. AIDS Res Hum Retroviruses 2019. 
392 9. Clutter DS, Zhou S, Varghese V, et al. Prevalence of Drug-Resistant Minority Variants in

393 Untreated HIV-1-Infected Individuals With and Those Without Transmitted Drug Resistance

394 Detected by Sanger Sequencing. J Infect Dis 2017; 216:387-91.

395 10. Dauwe K, Staelens D, Vancoillie L, Mortier V, Verhofstede C. Deep Sequencing of HIV-1 RNA

396 and DNA in Newly Diagnosed Patients with Baseline Drug Resistance Showed No Indications for

397 Hidden Resistance and Is Biased by Strong Interference of Hypermutation. J Clin Microbiol

398 2016; 54:1605-15.

399 11. Noguera-Julian M, Cozzi-Lepri A, Di Giallonardo F, et al. Contribution of APOBEC3G/F activity

400 to the development of low-abundance drug-resistant human immunodeficiency virus type 1

401 variants. Clin Microbiol Infect 2016; 22:191-200.

402 12. Bruner KM, Murray AJ, Pollack RA, et al. Defective proviruses rapidly accumulate during

403 acute HIV-1 infection. Nat Med 2016; 22:1043-9.

404 13. Delviks-Frankenberry KA, Nikolaitchik OA, Burdick RC, et al. Minimal Contribution of

405 APOBEC3-Induced G-to-A Hypermutation to HIV-1 Recombination and Genetic Variation. PLoS

406 Pathog 2016; 12:e1005646.

407 14. Taylor T, Lee ER, Nykoluk M, et al. A MiSeq-HyDRA platform for enhanced HIV drug

408 resistance genotyping and surveillance. Sci Rep 2019; 9:8970.

409 15. Langmead B, Salzberg SL. Fast gapped-read alignment with Bowtie 2. Nat Methods 2012;

$410 \quad 9: 357-9$.

411 16. Los Alamos National Laboratories. HIV Sequence Database: HIV Sequence Alignments. 
413 17. Rose PP, Korber BT. Detecting hypermutations in viral sequences with an emphasis on G -->

414 A hypermutation. Bioinformatics 2000; 16:400-1.

415 18. Jair K, McCann CD, Reed H, et al. Validation of publicly-available software used in analyzing

416 NGS data for HIV-1 drug resistance mutations and transmission networks in a Washington, DC,

417 Cohort. PLoS One 2019; 14:e0214820.

418 19. Moscona R, Ram D, Wax M, et al. Comparison between next-generation and Sanger-based

419 sequencing for the detection of transmitted drug-resistance mutations among recently infected

420 HIV-1 patients in Israel, 2000-2014. J Int AIDS Soc 2017; 20:21846.

421 20. Nguyen T, Fofana DB, Le MP, et al. Prevalence and clinical impact of minority resistant

422 variants in patients failing an integrase inhibitor-based regimen by ultra-deep sequencing. J

423 Antimicrob Chemother 2018; 73:2485-92.

424 21. Ode H, Matsuda M, Matsuoka K, et al. Quasispecies Analyses of the HIV-1 Near-full-length

425 Genome With Illumina MiSeq. Front Microbiol 2015; 6:1258.

426 22. Telele NF, Kalu AW, Gebre-Selassie S, et al. Pretreatment drug resistance in a large

427 countrywide Ethiopian HIV-1C cohort: a comparison of Sanger and high-throughput sequencing.

428 Sci Rep 2018; 8:7556.

429 23. Avila-Rios S, Garcia-Morales C, Matias-Florentino M, et al. HIV Drug Resistance in

430 Antiretroviral Treatment-Naive Individuals in the Largest Public Hospital in Nicaragua, 2011-

431 2015. PLoS One 2016; 11:e0164156.

432 24. Huber M, Metzner KJ, Geissberger FD, et al. MinVar: A rapid and versatile tool for HIV-1

433 drug resistance genotyping by deep sequencing. J Virol Methods 2017; 240:7-13. 
434 25. Dalmat RR, Makhsous N, Pepper GG, et al. Limited Marginal Utility of Deep Sequencing for

435 HIV Drug Resistance Testing in the Age of Integrase Inhibitors. J Clin Microbiol 2018; 56.

436 26. Jabara CB, Jones CD, Roach J, Anderson JA, Swanstrom R. Accurate sampling and deep

437 sequencing of the HIV-1 protease gene using a Primer ID. Proc Natl Acad Sci U S A 2011;

$438 \quad 108: 20166-71$.

439 27. Varghese V, Wang E, Babrzadeh F, et al. Nucleic acid template and the risk of a PCR-Induced

440 HIV-1 drug resistance mutation. PLoS One 2010; 5:e10992.

441 28. Macalalad AR, Zody MC, Charlebois $P$, et al. Highly sensitive and specific detection of rare

442 variants in mixed viral populations from massively parallel sequence data. PLoS Comput Biol

$443 \quad 2012 ; 8: e 1002417$. 


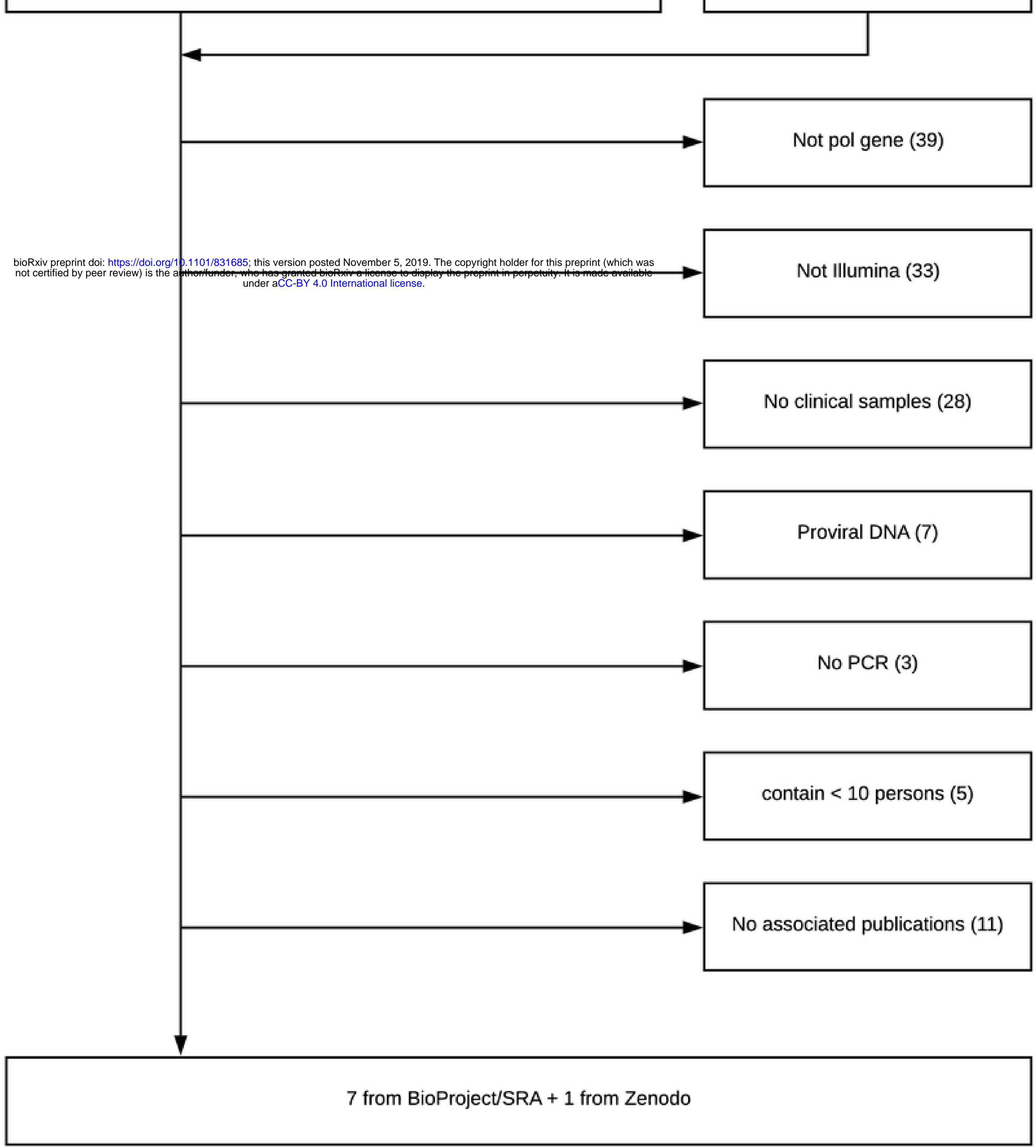

Figure 1 


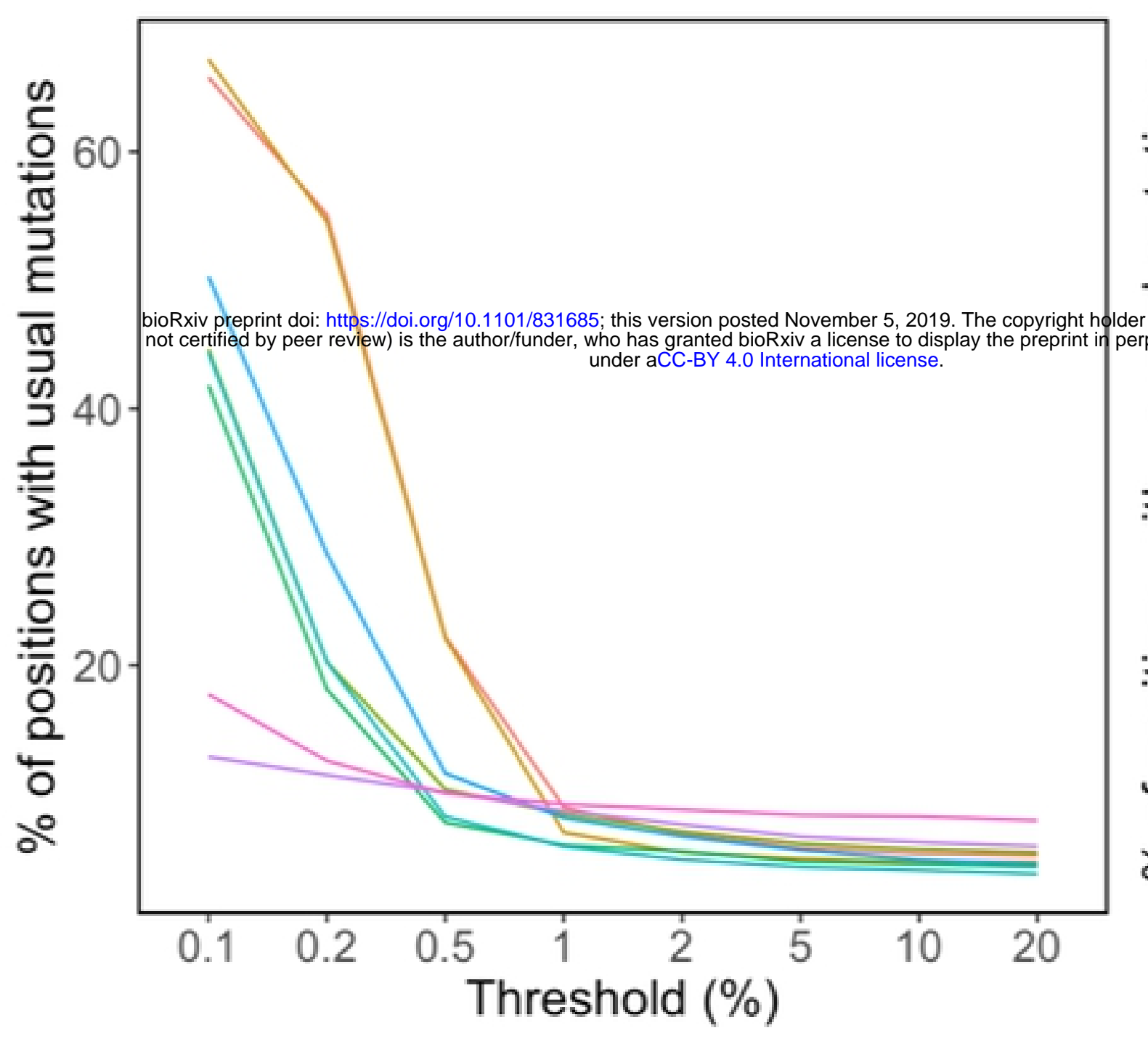

C

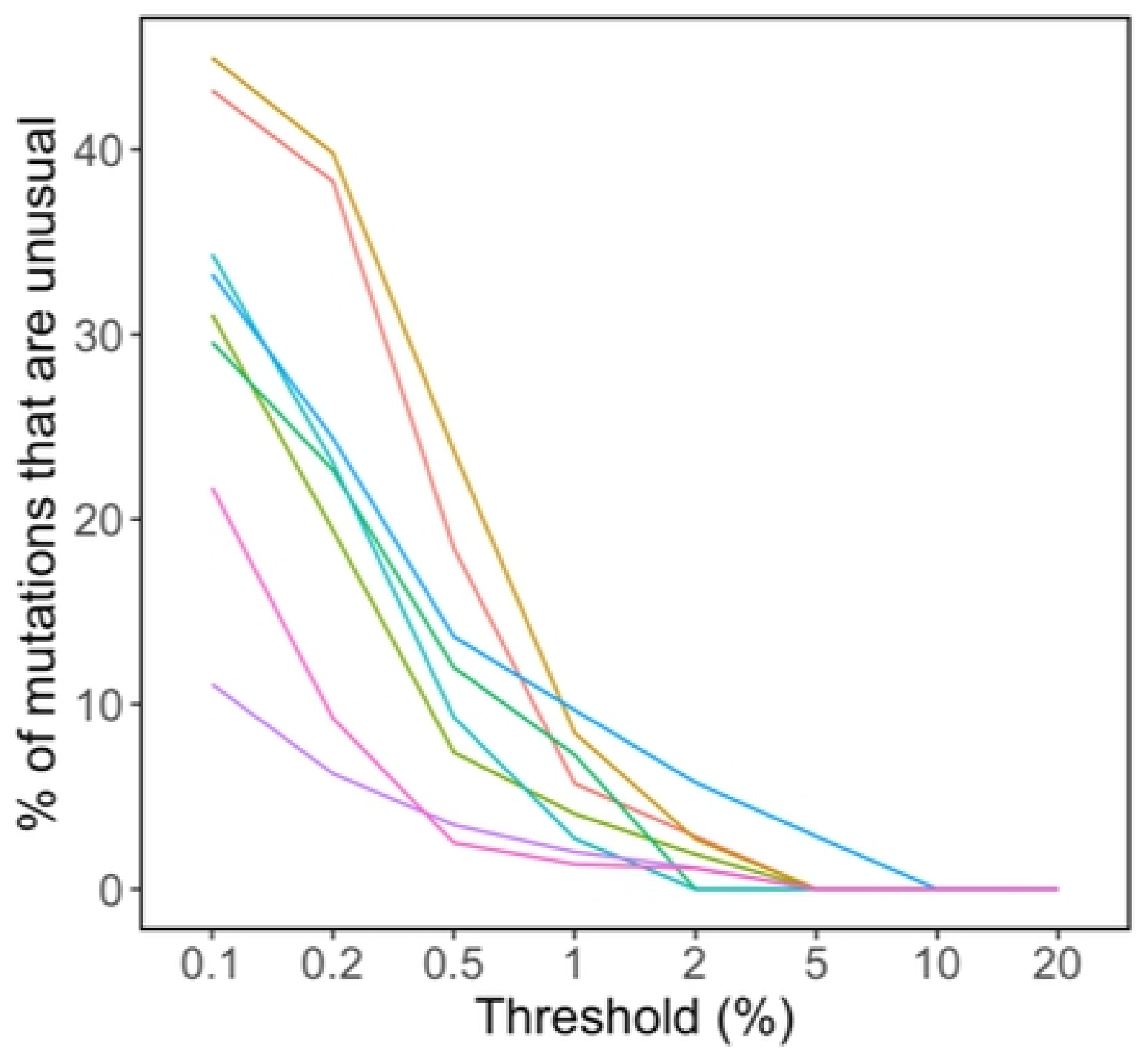

Figure 3 


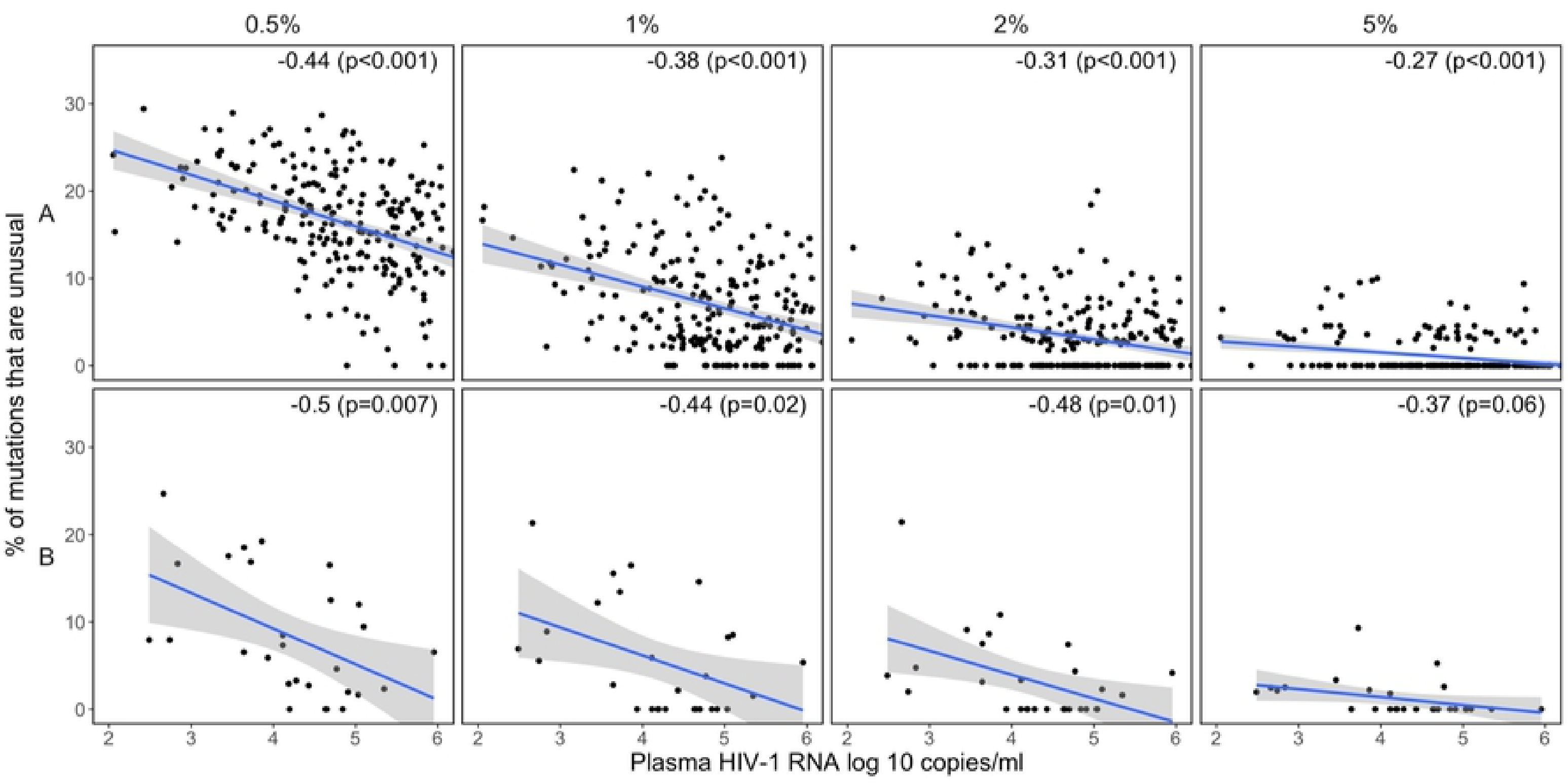

Figure 4 


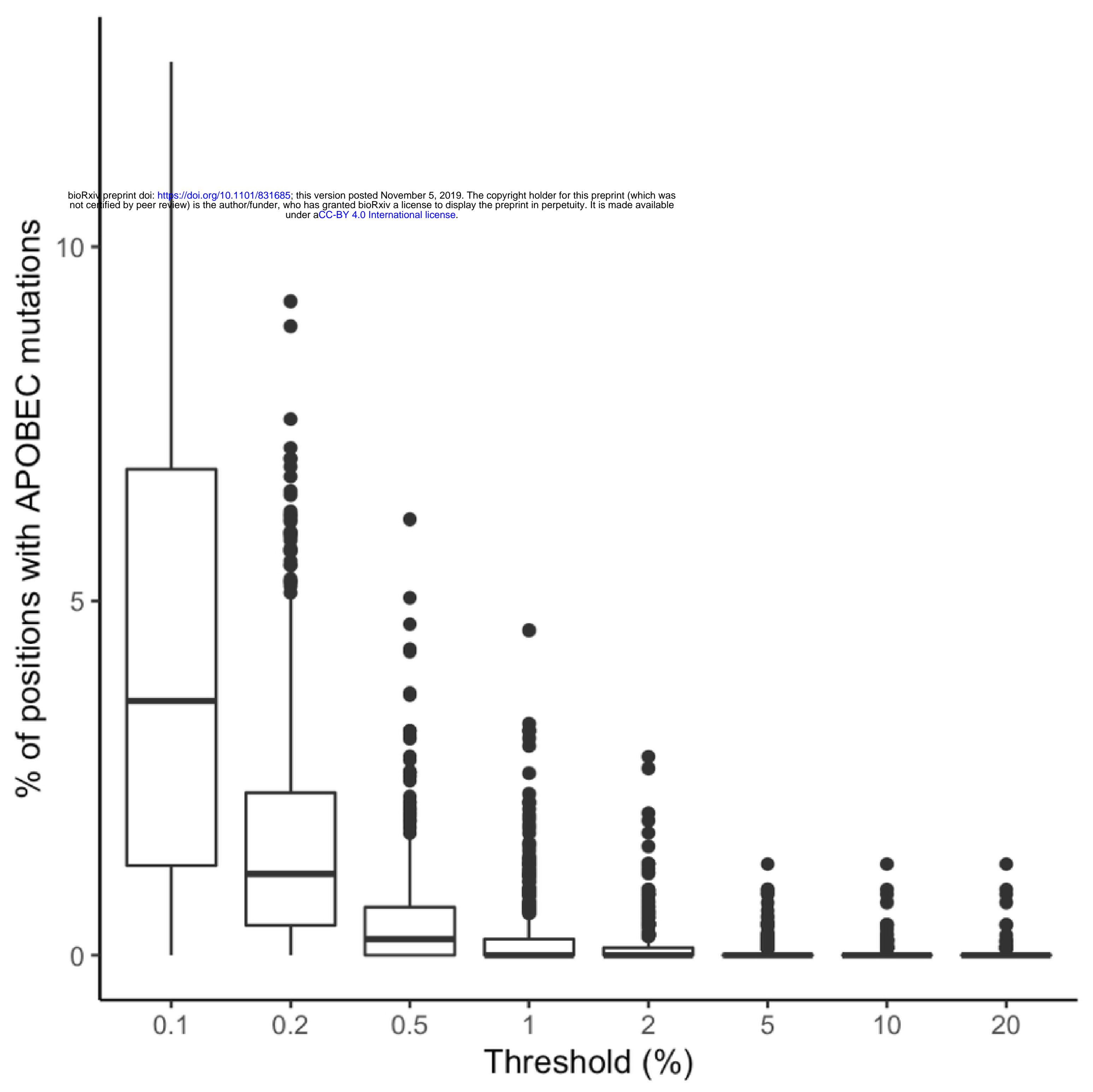

Figure 5 

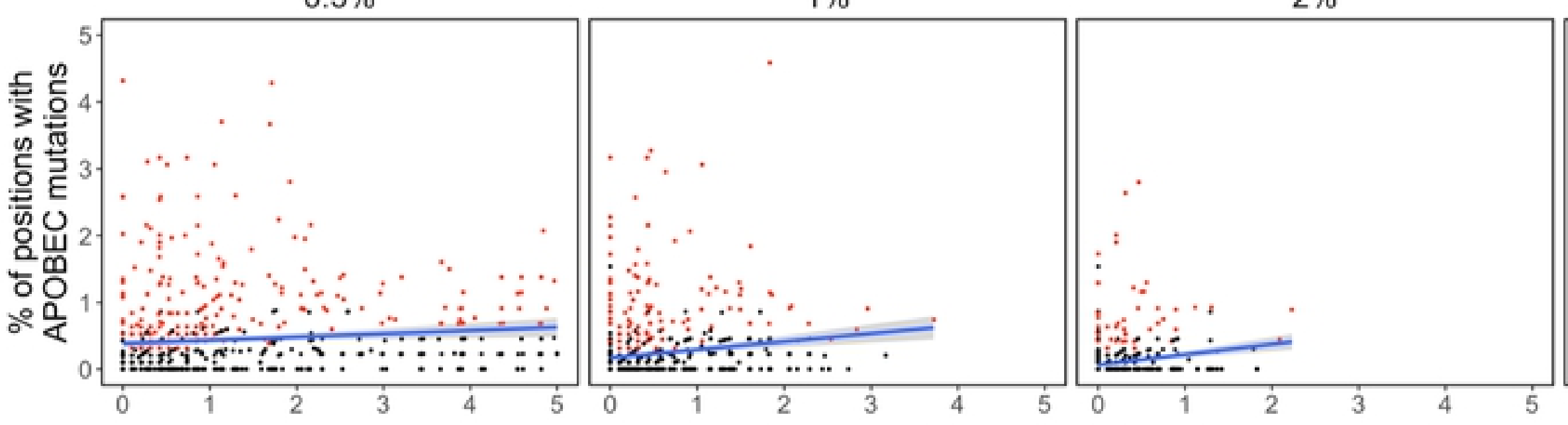

$\therefore \quad \therefore$

$\%$ of positions with Non-APOBEC unusual mutations

Figure 6 